\title{
Arithmetic properties for cubic partition pairs modulo powers of 3
}

\author{
Shane Chern
}

\begin{abstract}
Let $b(n)$ denote the number of cubic partition pairs of $n$. In this paper, we aim to provide a strategy to obtain arithmetic properties of $b(n)$. This gives affirmative answers to two of Lin's conjectures.
\end{abstract}

Keywords. Cubic partition pair, congruence.

2010MSC. Primary 05A17; Secondary 11P83.

\section{Introduction and main results}

A partition of a natural number $n$ is a nonincreasing sequence of positive integers whose sum equals $n$. Let $p(n)$ be the number of partitions. One of Ramanujan's most beautiful work is the following three congruences

$$
\begin{aligned}
p(5 n+4) \equiv 0 & (\bmod 5), \\
p(7 n+5) \equiv 0 & (\bmod 7), \\
p(11 n+6) & \equiv 0 \quad(\bmod 11) .
\end{aligned}
$$

Motivated by Ramanujan's result, Chan [2] introduced the cubic partition function $a(n)$ with generating function given by

$$
\sum_{n \geq 0} a(n) q^{n}=\frac{1}{(q ; q)_{\infty}\left(q^{2} ; q^{2}\right)_{\infty}}, \quad|q|<1,
$$

where and in the sequel, we use the standard notation

$$
(a ; q)_{\infty}=\prod_{n \geq 0}\left(1-a q^{n}\right) .
$$

One of the analogous congruences to Ramanujan's result is

$$
a(3 n+2) \equiv 0 \quad(\bmod 3),
$$

which can be deduced easily from the following identity obtained by Chan

$$
\sum_{n \geq 0} a(3 n+2) q^{n}=3 \frac{\left(q^{3} ; q^{3}\right)_{\infty}^{3}\left(q^{6} ; q^{6}\right)_{\infty}^{3}}{(q ; q)_{\infty}^{4}\left(q^{2} ; q^{2}\right)_{\infty}^{4}}
$$

Later on, many authors studied other Ramanujan-like congruences for $a(n)$. For instance, Chen and Lin [3] found four new congruences modulo 7, while recently the author and Dastidar [4] obtained two congruences modulo 11.

After Chan's work, many authors also investigated analogous partition functions. For instance, in 2011, Zhao and Zhong [8] studied the cubic partition pair function 
$b(n)$ given by

$$
\sum_{n \geq 0} b(n) q^{n}=\frac{1}{(q ; q)_{\infty}^{2}\left(q^{2} ; q^{2}\right)_{\infty}^{2}} .
$$

They obtained several congruences similar to Ramanujan's result:

$$
\begin{aligned}
b(5 n+4) & \equiv 0 \quad(\bmod 5), \\
b(7 n+i) & \equiv 0 \quad(\bmod 7), \\
b(9 n+7) & \equiv 0 \quad(\bmod 9),
\end{aligned}
$$

where $i=2,3,4,6$. For combinatorial proofs of the first two congruences, the reader may refer to Kim [5] and Zhou [9] respectively. In a recent paper, Lin [6] studied the arithmetic properties of $b(n)$ modulo 27. For instance, he showed the following infinite families of congruences

$$
b\left(81\left(7^{2 \alpha+1}(7 n+k)+\frac{7^{2 \alpha+2}-1}{12}\right)+7\right) \equiv 0 \quad(\bmod 27)
$$

for $\alpha \geq 0$ and $k=1,2,3,4,5,6$. He also conjectured the following three congruences modulo powers of 3 .

$$
b(81 n+61) \equiv 0 \quad(\bmod 243),
$$

and

$$
\begin{aligned}
\sum_{n \geq 0} b(81 n+7) q^{n} & \equiv 9 \frac{\left(q^{2} ; q^{2}\right)_{\infty}\left(q^{3} ; q^{3}\right)_{\infty}^{2}}{\left(q^{6} ; q^{6}\right)_{\infty}} \quad(\bmod 81), \\
\sum_{n \geq 0} b(81 n+34) q^{n} & \equiv 36 \frac{(q ; q)_{\infty}\left(q^{6} ; q^{6}\right)_{\infty}^{2}}{\left(q^{3} ; q^{3}\right)_{\infty}} \quad(\bmod 81) .
\end{aligned}
$$

In this paper, we will provide a strategy to give affirmative answers to Lin's conjectures. In fact, for (1.5), we have a stronger result

Theorem 1.1. For any $n \geq 0$,

$$
b(81 n+61) \equiv 0 \quad(\bmod 729) .
$$

We also obtain

Theorem 1.2. For any $n \geq 0$,

$$
b(243 n+61) \equiv 0 \quad(\bmod 2187) .
$$

For the remaining two congruences, we show

Theorem 1.3. Eqs. (1.6) and (1.7) hold for any $n \geq 0$.

\section{Preliminary results}

We first introduce two Ramanujan's theta functions $\varphi(q)$ and $\psi(q)$. They are defined by

$$
\begin{aligned}
& \varphi(q)=\frac{\left(q^{2} ; q^{2}\right)_{\infty}^{5}}{(q ; q)_{\infty}^{2}\left(q^{4} ; q^{4}\right)_{\infty}^{2}}=\sum_{n=-\infty}^{\infty} q^{n^{2}} \\
& \psi(q)=\frac{\left(q^{2} ; q^{2}\right)_{\infty}^{2}}{(q ; q)_{\infty}}=\sum_{n \geq 0} q^{n(n+1) / 2}
\end{aligned}
$$


Let

$$
w(q)=\frac{(q ; q)_{\infty}\left(q^{6} ; q^{6}\right)_{\infty}^{3}}{\left(q^{2} ; q^{2}\right)_{\infty}\left(q^{3} ; q^{3}\right)_{\infty}^{3}}
$$

In two recent papers, Zhao and Zhong [8], and Baruah and Ojah [1] obtained the 3 -dissection of $\varphi(-q)$ and $1 / \varphi(-q)$ respectively. Their results are

Lemma 2.1. It holds that

$$
\varphi(-q)=\varphi\left(-q^{9}\right)\left(1-2 q w\left(q^{3}\right)\right) .
$$

Lemma 2.2. It holds that

$$
\frac{1}{\varphi(-q)}=\frac{\varphi\left(-q^{9}\right)^{3}}{\varphi\left(-q^{3}\right)^{4}}\left(1+2 q w\left(q^{3}\right)+\left(2 q w\left(q^{3}\right)\right)^{2}\right) .
$$

For convenience, we write $\xi(q):=2 q w\left(q^{3}\right)$. Multiplying (2.1) and (2.2), we have

$$
\frac{\varphi\left(-q^{9}\right)^{4}}{\varphi\left(-q^{3}\right)^{4}}\left(1-\left(2 q w\left(q^{3}\right)\right)^{3}\right)=1
$$

It follows that

$$
\xi(q)^{3}=1-\frac{\varphi\left(-q^{3}\right)^{4}}{\varphi\left(-q^{9}\right)^{4}} .
$$

We also notice that in [7], Shen introduced 24 identities involving Ramanujan's theta functions. His Eq. (3.2) states

Lemma 2.3. It holds that

$$
\frac{\psi\left(q^{3}\right)^{3}}{\psi(q)}=\frac{1}{8 q}\left(\frac{\varphi\left(-q^{3}\right)^{3}}{\varphi(-q)}-\frac{\varphi(-q)^{3}}{\varphi\left(-q^{3}\right)}\right) .
$$

By Lemmas 2.1 and 2.2, we can rewrite it in the following form which will be frequently used in our proof:

$$
\begin{aligned}
\frac{1}{\psi(q)} & =\frac{1}{8 q \psi\left(q^{3}\right)^{3}}\left(\frac{\varphi\left(-q^{3}\right)^{3}}{\varphi(-q)}-\frac{\varphi(-q)^{3}}{\varphi\left(-q^{3}\right)}\right) \\
& =\frac{1}{8 q \psi\left(q^{3}\right)^{3}}\left(\frac{\varphi\left(-q^{9}\right)^{3}}{\varphi\left(-q^{3}\right)}\left(1+\xi(q)+\xi(q)^{2}\right)-\frac{\varphi\left(-q^{9}\right)^{3}}{\varphi\left(-q^{3}\right)}(1-\xi(q))^{3}\right) \\
& =\frac{1}{8 q \psi\left(q^{3}\right)^{3}} \frac{\varphi\left(-q^{9}\right)^{3}}{\varphi\left(-q^{3}\right)}\left(4 \xi(q)-2 \xi(q)^{2}+\xi(q)^{3}\right) .
\end{aligned}
$$

Given a series $\sum_{n \geq 0} u(n) q^{n}$, we say it is $(s, t, \lambda ; 4)$-expressible if we can find $\alpha \in \mathbb{Z}, \beta \in \mathbb{Z}_{>0}$ with $\beta \equiv \lambda(\bmod 4)$, and three finite sequences $\left(s_{1}, s_{2}, \ldots, s_{n}\right) \in$ $\mathbb{Z}^{n},\left(t_{1}, t_{2}, \ldots, t_{n}\right) \in \mathbb{Z}^{n},\left(v_{1}, v_{2}, \ldots, v_{n}\right):=\left(v\left(s_{1}, t_{1}\right), v\left(s_{2}, t_{2}\right), \ldots, v\left(s_{n}, t_{n}\right)\right) \in \mathbb{Q}^{n}$ with $s_{1} \equiv s_{2} \equiv \cdots \equiv s_{n} \equiv s(\bmod 4), t_{1} \equiv t_{2} \equiv \cdots \equiv t_{n} \equiv t(\bmod 4)$, and $s_{1}+t_{1}=s_{2}+t_{2}=\cdots=s_{n}+t_{n}$, such that

$$
\sum_{n \geq 0} u(n) q^{n}=\frac{q^{\alpha}}{\psi(q)^{\beta}} \sum_{i=1}^{n} v\left(s_{i}, t_{i}\right) \varphi(-q)^{s_{i}} \varphi\left(-q^{3}\right)^{t_{i}}
$$

The 3 -dissection of a $(s, t, \lambda ; 4)$-expressible series has the following property:

Lemma 2.4. If $s, t$, and $\lambda$ satisfy one of the following conditions:

(1) $\lambda=0$, and $s \equiv t(\bmod 4)$;

(2) $\lambda=2$, and $s \equiv t+2(\bmod 4)$, 
then given an $(s, t, \lambda ; 4)$-expressible series $\sum_{n \geq 0} u(n) q^{n}$, we can find an integer $\ell \in\{0,1,2\}$ such that $\sum_{n \geq 0} u(3 n+\ell) q^{n}$ is also $(s, t, \lambda ; 4)$-expressible.

Proof. If $\sum_{n \geq 0} u(n) q^{n}$ is $(a, b, \lambda ; 4)$-expressible, we can write it as

$$
\sum_{n \geq 0} u(n) q^{n}=\frac{q^{\alpha}}{\psi(q)^{\beta}} \sum_{i=1}^{n} v\left(s_{i}, t_{i}\right) \varphi(-q)^{s_{i}} \varphi\left(-q^{3}\right)^{t_{i}}
$$

By (2.5), we have

$$
\sum_{n \geq 0} u(n) q^{n}=\frac{q^{\alpha-\beta}}{8^{\beta} \psi\left(q^{3}\right)^{3 \beta}} \sum_{n \geq 0} g(n) q^{n}
$$

where

$$
\sum_{n \geq 0} g(n) q^{n}=\left(\frac{\varphi\left(-q^{9}\right)^{3}}{\varphi\left(-q^{3}\right)}\right)^{\beta}\left(4 \xi(q)-2 \xi(q)^{2}+\xi(q)^{3}\right)^{\beta} \sum_{i=1}^{n} v\left(s_{i}, t_{i}\right) \varphi(-q)^{s_{i}} \varphi\left(-q^{3}\right)^{t_{i}}
$$

Suppose that $\alpha-\beta \equiv \ell(\bmod 3)$, we have

$$
\sum_{n \geq 0} u(3 n+\ell) q^{n}=\frac{q^{(\alpha-\beta-\ell) / 3}}{8^{\beta} \psi(q)^{3 \beta}} \sum_{n \geq 0} g(3 n) q^{n} .
$$

Here note that if $\beta \equiv \lambda=0$ or $2(\bmod 4)$, we have $3 \beta \equiv \lambda(\bmod 4)$.

Next for any $i=1, \ldots, n$, we aim to obtain the 3 -dissection of

$$
\sum_{n \geq 0} g_{i}(n) q^{n}=\varphi(-q)^{s_{i}} \varphi\left(-q^{3}\right)^{t_{i}}\left(\frac{\varphi\left(-q^{9}\right)^{3}}{\varphi\left(-q^{3}\right)}\right)^{\beta}\left(4 \xi(q)-2 \xi(q)^{2}+\xi(q)^{3}\right)^{\beta} .
$$

If $s_{i} \geq 0$, then by Lemma 2.1,

$$
\begin{aligned}
& \varphi(-q)^{s_{i}} \varphi\left(-q^{3}\right)^{t_{i}}\left(\frac{\varphi\left(-q^{9}\right)^{3}}{\varphi\left(-q^{3}\right)}\right)^{\beta}\left(4 \xi(q)-2 \xi(q)^{2}+\xi(q)^{3}\right)^{\beta} \\
& \quad=\varphi\left(-q^{3}\right)^{t_{i}-\beta} \varphi\left(-q^{9}\right)^{s_{i}+3 \beta}(1-\xi(q))^{s_{i}}\left(4 \xi(q)-2 \xi(q)^{2}+\xi(q)^{3}\right)^{\beta} .
\end{aligned}
$$

Now we pick up terms of the form $q^{3 n}$ and obtain

$$
\varphi\left(-q^{3}\right)^{t_{i}-\beta} \varphi\left(-q^{9}\right)^{s_{i}+3 \beta} \sum_{j=0}^{\left\lfloor\frac{s_{i}+3 \beta}{3}\right\rfloor} m_{3 j} \xi(q)^{3 j}
$$

for some $m_{3 j}$. By (2.3), we therefore have

$$
\begin{aligned}
\sum_{n \geq 0} g_{i}(3 n) q^{n} & =\varphi(-q)^{t_{i}-\beta} \varphi\left(-q^{3}\right)^{s_{i}+3 \beta} \sum_{j=0}^{\left\lfloor\frac{s_{i}+3 \beta}{3}\right\rfloor} m_{3 j}\left(1-\frac{\varphi(-q)^{4}}{\varphi\left(-q^{3}\right)^{4}}\right)^{j} \\
& =\sum_{j=0}^{\left\lfloor\frac{s_{i}+3 \beta}{3}\right\rfloor} v^{\prime}\left(s_{i, j}^{\prime}, t_{i, j}^{\prime}\right) \varphi(-q)^{s_{i, j}^{\prime}} \varphi\left(-q^{3}\right)^{t_{i, j}^{\prime}},
\end{aligned}
$$

where

$$
\left\{\begin{array}{l}
s_{i, j}^{\prime}=t_{i}-\beta+4 j \\
t_{i, j}^{\prime}=s_{i}+3 \beta-4 j
\end{array}\right.
$$


for $j=0, \ldots,\left\lfloor\left(s_{i}+3 \beta\right) / 3\right\rfloor$. For both conditions (i) and (ii), it is easy to see that $s_{i, j}^{\prime} \equiv s_{i}(\bmod 4)$ and $t_{i, j}^{\prime} \equiv t_{i}(\bmod 4)$ for all $j$. Similarly, if $s_{j}<0$, we can write $\sum_{n \geq 0} g_{i}(3 n) q^{n}$ as a linear combination of $\varphi(-q)^{s_{i, j}^{\prime}} \varphi\left(-q^{3}\right)^{t_{i, j}^{\prime}}$ where

$$
\left\{\begin{array}{l}
s_{i, j}^{\prime}=t_{i}+4 s_{i}-\beta+4 j \\
t_{i, j}^{\prime}=-3 s_{i}+3 \beta-4 j
\end{array}\right.
$$

for $j=0, \ldots,\left\lfloor\left(-2 s_{i}+3 \beta\right) / 3\right\rfloor$. For both conditions (i) and (ii), we also see that $s_{i, j}^{\prime} \equiv s_{i}(\bmod 4)$ and $t_{i, j}^{\prime} \equiv t_{i}(\bmod 4)$ for all $j$. Furthermore, we have $s_{i, j}^{\prime}+t_{i, j}^{\prime}=$ $s_{i}+t_{i}+2 \beta$ for all $j$ whenever $s_{i} \geq 0$ or $s_{i}<0$.

Since the above argument holds for all $i=1, \ldots, n$, we therefore can find three new finite sequences $\left(s_{1}^{\prime}, s_{2}^{\prime}, \ldots, s_{n^{\prime}}^{\prime}\right) \in \mathbb{Z}^{n^{\prime}},\left(t_{1}^{\prime}, t_{2}^{\prime}, \ldots, t_{n^{\prime}}^{\prime}\right) \in \mathbb{Z}^{n^{\prime}},\left(v_{1}^{\prime}, v_{2}^{\prime}, \ldots, v_{n^{\prime}}^{\prime}\right):=$ $\left(v^{\prime}\left(s_{1}^{\prime}, t_{1}^{\prime}\right), v^{\prime}\left(s_{2}^{\prime}, t_{2}^{\prime}\right), \ldots, v^{\prime}\left(s_{n^{\prime}}^{\prime}, t_{n^{\prime}}^{\prime}\right)\right) \in \mathbb{Q}^{n^{\prime}}$ with $s_{1}^{\prime} \equiv s_{2}^{\prime} \equiv \cdots \equiv s_{n^{\prime}}^{\prime} \equiv s(\bmod 4)$, $t_{1}^{\prime} \equiv t_{2}^{\prime} \equiv \cdots \equiv t_{n^{\prime}}^{\prime} \equiv t(\bmod 4)$, and $s_{1}^{\prime}+t_{1}^{\prime}=s_{2}^{\prime}+t_{2}^{\prime}=\cdots=s_{n^{\prime}}^{\prime}+t_{n^{\prime}}^{\prime}$, such that

$$
\sum_{n \geq 0} u(3 n+\ell) q^{n}=\frac{q^{(\alpha-\beta-\ell) / 3}}{\psi(q)^{3 \beta}} \sum_{i=1}^{n^{\prime}} v^{\prime}\left(s_{i}^{\prime}, t_{i}^{\prime}\right) \varphi(-q)^{s_{i}^{\prime}} \varphi\left(-q^{3}\right)^{t_{i}^{\prime}} .
$$

Remark 2.1. The aim of this lemma is to show that if a series $\sum_{n \geq 0} u(n) q^{n}$ is $(s, t, \lambda ; 4)$-expressible satisfying conditions (i) or (ii), then we may write

$$
\sum_{n \geq 0} u\left(3^{k} n+\ell\right) q^{n}
$$

(for some computable $\ell$ ) as the form

$$
\frac{q^{\alpha}}{\psi(q)^{\beta}} \sum_{i=1}^{n} v\left(s_{i}, t_{i}\right) \varphi(-q)^{s_{i}} \varphi\left(-q^{3}\right)^{t_{i}}
$$

In this sense, we can study the arithmetic property of $u\left(3^{k} n+\ell\right)$ by studying the coefficients $v\left(s_{i}, t_{i}\right)$.

\section{Proofs}

In view of (1.4), we can rewrite the generating function of $b(n)$ as

$$
\sum_{n \geq 0} b(n) q^{n}=\frac{1}{\psi(q)^{2} \varphi(-q)^{2}}=\frac{\varphi\left(-q^{3}\right)^{0}}{\psi(q)^{2} \varphi(-q)^{2}} .
$$

Hence $\sum_{n \geq 0} b(n) q^{n}$ is $(2,0,2 ; 4)$-expressible. According to Lemma 2.4, we can see that for any positive integer $\alpha$, we can find some $\ell$, such that $\sum_{n \geq 0} b\left(3^{\alpha} n+\ell\right) q^{n}$ has the form

$$
\frac{q^{\alpha}}{\psi(q)^{\beta}} \sum_{i=1}^{n} v\left(s_{i}, t_{i}\right) \varphi(-q)^{s_{i}} \varphi\left(-q^{3}\right)^{t_{i}}
$$

where $s_{1} \equiv s_{2} \equiv \cdots \equiv s_{n} \equiv 2(\bmod 4), t_{1} \equiv t_{2} \equiv \cdots \equiv t_{n} \equiv 0(\bmod 4)$, and $s_{1}+t_{1}=s_{2}+t_{2}=\cdots=s_{n}+t_{n}$.

Using the method presented in the proof of Lemma 2.4, we first give the detail of the 3-dissection of $\sum_{n \geq 0} b(n) q^{n}$.

$$
\frac{1}{\psi(q)^{2} \varphi(-q)^{2}}=\frac{q^{-2}}{2^{6} \psi\left(q^{3}\right)^{6}}\left(\frac{\varphi\left(-q^{9}\right)^{3}}{\varphi\left(-q^{3}\right)}\right)^{2}\left(4 \xi(q)-2 \xi(q)^{2}+\xi(q)^{3}\right)^{2}
$$




$$
\begin{aligned}
& \times\left(\frac{\varphi\left(-q^{9}\right)^{3}}{\varphi\left(-q^{3}\right)^{4}}\right)^{2}\left(1+\xi(q)+\xi(q)^{2}\right)^{2} \\
= & \frac{q^{-3} \cdot q}{2^{6} \psi\left(q^{3}\right)^{6}} \frac{\varphi\left(-q^{9}\right)^{12}}{\varphi\left(-q^{3}\right)^{10}}\left(4 \xi(q)-2 \xi(q)^{2}+\xi(q)^{3}\right)^{2}\left(1+\xi(q)+\xi(q)^{2}\right)^{2} .
\end{aligned}
$$

Extracting terms involving $q^{3 n+1}$ and replacing $q^{3 n}$ by $q^{n}$, we have

$$
\sum_{n \geq 0} b(3 n+1) q^{n}=\frac{q^{-1}}{2^{6} \psi(q)^{6}}\left(2 \varphi(-q)^{2}+7 \frac{\varphi\left(-q^{3}\right)^{4}}{\varphi(-q)^{2}}-36 \frac{\varphi\left(-q^{3}\right)^{8}}{\varphi(-q)^{6}}+27 \frac{\varphi\left(-q^{3}\right)^{12}}{\varphi(-q)^{10}}\right) .
$$

Using the same method, we can obtain

$$
\begin{aligned}
& \sum_{n \geq 0} b(9 n+7) q^{n} \\
&=\frac{q^{-3}}{2^{24} \psi(q)^{18}}\left(252 \frac{\varphi(-q)^{18}}{\varphi\left(-q^{3}\right)^{4}}+16254 \varphi(-q)^{14}+54054 \varphi(-q)^{10} \varphi\left(-q^{3}\right)^{4}\right. \\
&+54180 \varphi(-q)^{6} \varphi\left(-q^{3}\right)^{8}-3679992 \varphi(-q)^{2} \varphi\left(-q^{3}\right)^{12}+33485805 \frac{\varphi\left(-q^{3}\right)^{16}}{\varphi(-q)^{2}} \\
&-201778452 \frac{\varphi\left(-q^{3}\right)^{20}}{\varphi(-q)^{6}}+846955116 \frac{\varphi\left(-q^{3}\right)^{24}}{\varphi(-q)^{10}}-2445337188 \frac{\varphi\left(-q^{3}\right)^{28}}{\varphi(-q)^{14}} \\
&+4746831012 \frac{\varphi\left(-q^{3}\right)^{32}}{\varphi(-q)^{18}}-6004220418 \frac{\varphi\left(-q^{3}\right)^{36}}{\varphi(-q)^{22}}+4706441496 \frac{\varphi\left(-q^{3}\right)^{40}}{\varphi(-q)^{26}} \\
&\left.-2066242608 \frac{\varphi\left(-q^{3}\right)^{44}}{\varphi(-q)^{30}}+387420489 \frac{\varphi\left(-q^{3}\right)^{48}}{\varphi(-q)^{34}}\right) .
\end{aligned}
$$

Checking the coefficients $252,16254, \ldots$, we can see that they are all divisible by 9. It therefore follows that

$$
b(9 n+7) \equiv 0 \quad(\bmod 9)
$$

for all $n \geq 0$, which was obtained previously by Zhao and Zhong [8].

For the following dissections, the coefficients become very large. Hence we only display them under modulus $2187=3^{7}$. We have

$$
\begin{aligned}
& \sum_{n \geq 0} b(27 n+7) q^{n} \\
& \equiv \frac{q^{-7}}{2^{78} \psi(q)^{54}}\left(252 \frac{\varphi(-q)^{74}}{\varphi\left(-q^{3}\right)^{24}}+504 \frac{\varphi(-q)^{70}}{\varphi\left(-q^{3}\right)^{20}}+918 \frac{\varphi(-q)^{66}}{\varphi\left(-q^{3}\right)^{16}}+2034 \frac{\varphi(-q)^{62}}{\varphi\left(-q^{3}\right)^{12}}\right. \\
&+396 \frac{\varphi(-q)^{58}}{\varphi\left(-q^{3}\right)^{8}}+1755 \frac{\varphi(-q)^{54}}{\varphi\left(-q^{3}\right)^{4}}+225 \varphi(-q)^{50}+1530 \varphi(-q)^{46} \varphi\left(-q^{3}\right)^{4} \\
&\left.+1701 \varphi(-q)^{42} \varphi\left(-q^{3}\right)^{8}+1620 \varphi(-q)^{38} \varphi\left(-q^{3}\right)^{12}\right) \quad(\bmod 2187)
\end{aligned}
$$

Continuing the dissection, we have

$$
\begin{aligned}
& \sum_{n \geq 0} b(81 n+61) q^{n} \\
& \equiv \frac{q^{-21}}{2^{240} \psi(q)^{162}}\left(729 \frac{\varphi(-q)^{230}}{\varphi\left(-q^{3}\right)^{72}}+1458 \frac{\varphi(-q)^{226}}{\varphi\left(-q^{3}\right)^{68}}+1458 \frac{\varphi(-q)^{218}}{\varphi\left(-q^{3}\right)^{60}}+729 \frac{\varphi(-q)^{214}}{\varphi\left(-q^{3}\right)^{56}}\right.
\end{aligned}
$$




$$
\begin{aligned}
& +729 \frac{\varphi(-q)^{194}}{\varphi\left(-q^{3}\right)^{36}}+1458 \frac{\varphi(-q)^{190}}{\varphi\left(-q^{3}\right)^{32}}+1458 \frac{\varphi(-q)^{182}}{\varphi\left(-q^{3}\right)^{24}}+729 \frac{\varphi(-q)^{178}}{\varphi\left(-q^{3}\right)^{20}} \\
& +729 \varphi(-q)^{158}+1458 \varphi(-q)^{154} \varphi\left(-q^{3}\right)^{4}+1458 \varphi(-q)^{146} \varphi\left(-q^{3}\right)^{12} \\
& \left.+729 \varphi(-q)^{142} \varphi\left(-q^{3}\right)^{16}\right) \quad(\bmod 2187) .
\end{aligned}
$$

Now by checking the coefficients modulo 729 , we can see that

$$
b(81 n+61) \equiv 0 \quad(\bmod 729),
$$

and thus complete the proof of Theorem 1.1.

Furthermore, we can rewrite $\sum_{n \geq 0} b(81 n+61) q^{n}$ as

$$
\begin{aligned}
\sum_{n \geq 0} b(81 n+61) q^{n} & \\
\equiv & \frac{729 q^{-21}}{2^{240} \psi(q)^{162}} \varphi(-q)^{142} \varphi\left(-q^{3}\right)^{16} \\
& \quad \times\left(1+\kappa(q)^{9}+\kappa(q)^{18}\right)\left(1+2 \kappa(q)+2 \kappa(q)^{3}+\kappa(q)^{4}\right) \quad(\bmod 2187),
\end{aligned}
$$

where

According to (2.3), we have

$$
\kappa(q)=\frac{\varphi(-q)^{4}}{\varphi\left(-q^{3}\right)^{4}}
$$

$$
\kappa(q)=1-8 q w(q)^{3}
$$

and thus

$$
\kappa(q) \equiv 1-2 q w\left(q^{3}\right)=1-\xi(q) \quad(\bmod 3) .
$$

To prove Theorem 1.2, we note that

$$
\begin{aligned}
& \frac{q^{-21}}{2^{240} \psi(q)^{162}} \varphi(-q)^{142} \varphi\left(-q^{3}\right)^{16}\left(1+\kappa(q)^{9}+\kappa(q)^{18}\right)\left(1+2 \kappa(q)+2 \kappa(q)^{3}+\kappa(q)^{4}\right) \\
& \equiv \frac{\left(q^{3}\right)^{-7}}{\psi\left(q^{3}\right)^{54}}\left(\varphi(-q) \varphi\left(-q^{3}\right)^{47}\right) \varphi\left(-q^{3}\right)^{16}\left(1+\kappa\left(q^{3}\right)^{3}+\kappa\left(q^{3}\right)^{6}\right)(1-\kappa(q))^{4} \\
& \equiv \frac{\left(q^{3}\right)^{-7}}{\psi\left(q^{3}\right)^{54}} \varphi\left(-q^{3}\right)^{63} \varphi\left(-q^{9}\right)\left(1+\kappa\left(q^{3}\right)^{3}+\kappa\left(q^{3}\right)^{6}\right)(1-\xi(q)) \xi(q)^{4} \quad(\bmod 3) .
\end{aligned}
$$

Hence

$$
\begin{aligned}
& \sum_{n \geq 0} b(81 n+61) q^{n} \\
& \quad \equiv \frac{729\left(q^{3}\right)^{-7}}{\psi\left(q^{3}\right)^{54}} \varphi\left(-q^{3}\right)^{63} \varphi\left(-q^{9}\right)\left(1+\kappa\left(q^{3}\right)^{3}+\kappa\left(q^{3}\right)^{6}\right)(1-\xi(q)) \xi(q)^{4} \quad(\bmod 2187) .
\end{aligned}
$$

Since $\xi(q)=2 q w\left(q^{3}\right)$, we can see that the right hand side contains no term of the form $q^{3 n}$. Hence

$$
b(243 n+61) \equiv 0 \quad(\bmod 2187)
$$

holds for all $n \geq 0$.

We next prove Theorem 1.3. If we extract terms of the form $q^{3 n}$ from $\sum_{n \geq 0} b(27 n+$ 7) $q^{n}$ and replace $q^{3}$ by $q$, we have

$$
\sum_{n \geq 0} b(81 n+7) q^{n}
$$




$$
\begin{aligned}
& \equiv \frac{9 q^{-20} w(q) \varphi(-q)^{154} \varphi\left(-q^{3}\right)^{4}}{2^{239} \psi(q)^{162}} \\
& \times\left(5+8 \kappa(q)+5 \kappa(q)^{2}+6 \kappa(q)^{3}+6 \kappa(q)^{4}+6 \kappa(q)^{5}+3 \kappa(q)^{6}\right. \\
& \quad+3 \kappa(q)^{7}+3 \kappa(q)^{8}+8 \kappa(q)^{9}+2 \kappa(q)^{10}+8 \kappa(q)^{11}+3 \kappa(q)^{12} \\
& \quad+3 \kappa(q)^{13}+3 \kappa(q)^{14}+6 \kappa(q)^{15}+6 \kappa(q)^{16}+6 \kappa(q)^{17}+5 \kappa(q)^{18} \\
& \left.\quad+8 \kappa(q)^{19}+5 \kappa(q)^{20}\right)(\bmod 81) .
\end{aligned}
$$

Note that

$$
\begin{aligned}
(1-x)^{20} \equiv 1 & +7 x+x^{2}+3 x^{3}+3 x^{4}+3 x^{5}+6 x^{6}+6 x^{7}+6 x^{8} \\
& +7 x^{9}+4 x^{10}+7 x^{11}+6 x^{12}+6 x^{13}+6 x^{14}+3 x^{15}+3 x^{16} \\
& +3 x^{17}+x^{18}+7 x^{19}+x^{20} \quad(\bmod 9) .
\end{aligned}
$$

We therefore have

$$
\begin{aligned}
& \sum_{n \geq 0} b(81 n+7) q^{n} \\
& \quad \equiv 9 \times \frac{5 q^{-20} w(q) \varphi(-q)^{154} \varphi\left(-q^{3}\right)^{4}}{2^{239} \psi(q)^{162}}(1-\kappa(q))^{20} \\
& \quad \equiv 9 \frac{(q ; q)_{\infty}^{531}\left(q^{6} ; q^{6}\right)_{\infty}^{179}}{\left(q^{2} ; q^{2}\right)_{\infty}^{539}\left(q^{3} ; q^{3}\right)_{\infty}^{175}}(\bmod 81) .
\end{aligned}
$$

One readily sees from the binomial theorem that

$$
(q ; q)_{\infty}^{9} \equiv\left(q^{3} ; q^{3}\right)_{\infty}^{3} \quad(\bmod 9)
$$

We therefore conclude that

$$
\begin{aligned}
& \sum_{n \geq 0} b(81 n+7) q^{n} \\
& \quad \equiv 9\left(q^{3} ; q^{3}\right)_{\infty}^{2}\left(\frac{(q ; q)_{\infty}^{9}}{\left(q^{3} ; q^{3}\right)_{\infty}^{3}}\right)^{59} \frac{\left(q^{2} ; q^{2}\right)_{\infty}}{\left(q^{6} ; q^{6}\right)_{\infty}}\left(\frac{\left(q^{6} ; q^{6}\right)_{\infty}^{3}}{\left(q^{2} ; q^{2}\right)_{\infty}^{9}}\right)^{60} \\
& \quad \equiv 9 \frac{\left(q^{2} ; q^{2}\right)_{\infty}\left(q^{3} ; q^{3}\right)_{\infty}^{2}}{\left(q^{6} ; q^{6}\right)_{\infty}} \quad(\bmod 81) .
\end{aligned}
$$

This proves (1.6).

At last, we extract terms of the form $q^{3 n+1}$ from $\sum_{n \geq 0} b(27 n+7) q^{n}$ and replace $q^{3}$ by $q$. Then

$$
\begin{aligned}
& \sum_{n \geq 0} b(81 n+34) q^{n} \\
& \equiv 9 \frac{q^{-20} w(q)^{2} \varphi(-q)^{154} \varphi\left(-q^{3}\right)^{4}}{2^{238} \psi(q)^{162}} \\
& \times\left(1+7 \kappa(q)+\kappa(q)^{2}+3 \kappa(q)^{3}+3 \kappa(q)^{4}+3 \kappa(q)^{5}+6 \kappa(q)^{6}\right. \\
& \quad+6 \kappa(q)^{7}+6 \kappa(q)^{8}+7 \kappa(q)^{9}+4 \kappa(q)^{10}+7 \kappa(q)^{11}+6 \kappa(q)^{12} \\
& +6 \kappa(q)^{13}+6 \kappa(q)^{14}+3 \kappa(q)^{15}+3 \kappa(q)^{16}+3 \kappa(q)^{17}+\kappa(q)^{18} \\
& \left.+7 \kappa(q)^{19}+\kappa(q)^{20}\right)
\end{aligned}
$$




$$
\begin{aligned}
& \equiv 9 \frac{q^{-20} w(q)^{2} \varphi(-q)^{154} \varphi\left(-q^{3}\right)^{4}}{2^{238} \psi(q)^{162}}(1-\kappa(q))^{20} \\
& \equiv 36 \frac{(q ; q)_{\infty}^{532}\left(q^{6} ; q^{6}\right)_{\infty}^{182}}{\left(q^{2} ; q^{2}\right)_{\infty}^{540}\left(q^{3} ; q^{3}\right)_{\infty}^{178}} \\
& \equiv 36 \frac{(q ; q)_{\infty}}{\left(q^{3} ; q^{3}\right)_{\infty}}\left(\frac{(q ; q)_{\infty}^{9}}{\left(q^{3} ; q^{3}\right)_{\infty}^{3}}\right)^{59}\left(q^{6} ; q^{6}\right)_{\infty}^{2}\left(\frac{\left(q^{6} ; q^{6}\right)_{\infty}^{3}}{\left(q^{2} ; q^{2}\right)_{\infty}^{9}}\right)^{60} \\
& \equiv 36 \frac{(q ; q)_{\infty}\left(q^{6} ; q^{6}\right)_{\infty}^{2}}{\left(q^{3} ; q^{3}\right)_{\infty}}(\bmod 81) .
\end{aligned}
$$

We therefore end the proof of (1.7).

\section{Concluding remarks}

Recall that from Lemma 2.4, for any positive integer $\alpha$, we can find some $\ell$, such that $\sum_{n \geq 0} b\left(3^{\alpha} n+\ell\right) q^{n}$ has the form

$$
\frac{q^{\alpha}}{\psi(q)^{\beta}} \sum_{i=1}^{n} v\left(s_{i}, t_{i}\right) \varphi(-q)^{s_{i}} \varphi\left(-q^{3}\right)^{t_{i}}
$$

where $s_{1} \equiv s_{2} \equiv \cdots \equiv s_{n} \equiv 2(\bmod 4), t_{1} \equiv t_{2} \equiv \cdots \equiv t_{n} \equiv 0(\bmod 4)$, and $s_{1}+t_{1}=s_{2}+t_{2}=\cdots=s_{n}+t_{n}$. In fact, it is easy to show by induction that

$$
\ell= \begin{cases}1+\frac{3^{\alpha+1}-3}{4} & \alpha \text { even } \\ 1+\frac{3^{\alpha}-3}{4} & \alpha \text { odd }\end{cases}
$$

From Theorem 1.2, we see that for $\alpha=6$ and 7 (and thus $\ell=547$ in both cases),

$$
b(729 n+547) \equiv 0 \quad(\bmod 2187)
$$

and

$$
b(2187 n+547) \equiv 0 \quad(\bmod 2187) .
$$

However the modulus $2187=3^{7}$ is the best choice since

$$
\begin{aligned}
b(547) & =2135474526556068875092854278074796547960 \\
& =2^{3} \times 3^{7} \times 5 \times 41 \times 61 \times 151 \times 11909 \times 5427748132276664632973303 .
\end{aligned}
$$

We therefore want to know if there is a general family of congruences modulo higher power of 3 for $b\left(3^{\alpha} n+\ell\right)$ ?

\section{Acknowledgement}

Many thanks go to George E. Andrews for encouraging me in the study of partition theory.

\section{References}

1. N. D. Baruah and K. K. Ojah, Some congruences deducible from Ramanujan's cubic continued fraction, Int. J. Number Theory 7 (2011), no. 5, 1331-1343.

2. H. C. Chan, Ramanujan's cubic continued fraction and an analog of his "most beautiful identity", Int. J. Number Theory 6 (2010), no. 3, 673-680.

3. W. Y. C. Chen and B. L. S. Lin, Congruences for the number of cubic partitions derived from modular forms, Preprint, arXiv:0910.1263, 15 pp.

4. S. Chern and M. G. Dastidar, Congruences and recursions for the cubic partition, to appear in Ramanujan J. 
5. B. Kim, Partition statistics for cubic partition pairs, Electron. J. Combin. 18 (2011), no. 1, Paper 128, 7 pp.

6. B. L. S. Lin, Congruences modulo 27 for cubic partition pairs, J. Number Theory 171 (2017), 31-42.

7. L. C. Shen, On the modular equations of degree 3, Proc. Amer. Math. Soc. 122 (1994), no. 4, 1101-1114.

8. H. Zhao and Z. Zhong, Ramanujan type congruences for a partition function, Electron. J. Combin. 18 (2011), no. 1, Paper 58, 9 pp.

9. R. R. Zhou, Multiranks for partitions into multi-colors, Electron. J. Combin. 19 (2012), no. 2, Paper 8, 17 pp.

Department of Mathematics, Pennsylvania State University, University Park, PA 16802, USA

E-mail address: shanechern@psu.edu; chenxiaohang92@gmail.com 